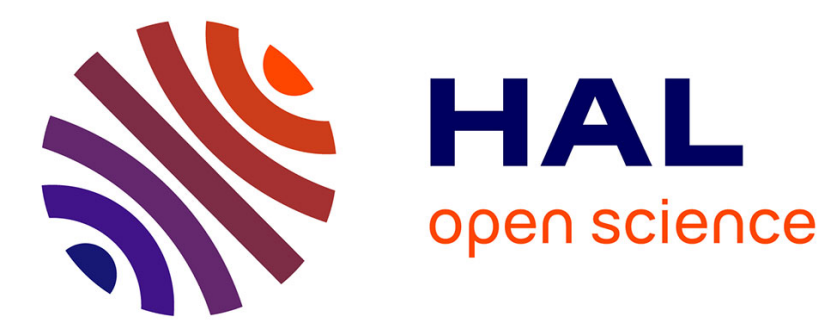

\title{
Evolution récente et perspectives de l'aviculture
}

P. Stevens

\section{- To cite this version:}

P. Stevens. Evolution récente et perspectives de l'aviculture. Productions Animales, 1988, 1 (3), pp.155-163. hal-00895827

\section{HAL Id: hal-00895827 \\ https://hal.science/hal-00895827}

Submitted on 1 Jan 1988

HAL is a multi-disciplinary open access archive for the deposit and dissemination of scientific research documents, whether they are published or not. The documents may come from teaching and research institutions in France or abroad, or from public or private research centers.
L'archive ouverte pluridisciplinaire HAL, est destinée au dépôt et à la diffusion de documents scientifiques de niveau recherche, publiés ou non, émanant des établissements d'enseignement et de recherche français ou étrangers, des laboratoires publics ou privés. 
INRA Prod. Anim., 1988, 1 (3), 155-163

\section{P. STEVENS}

INRA Nouzilly

Station de Recherches Avicoles

37380 Monnaie

\title{
Evolution récente et perspectives de l'aviculture
}

\author{
Sur un marché agricole encombré de produits en quête de débouchés \\ solvables, la consommation et l'exportation des produits avicoles \\ ne cessent de se développer... Actuellement, la France est le premier \\ producteur avicole de la C.E.E. avec $18 \%$ de la production communautaire \\ d'œufs et $23 \%$ de celle de viandes de volailles.
}

Placée dès 1962 dans un contexte communautaire qui interdisait toute intervention nationale, l'aviculture a évolué pour satisfaire au mieux les exigences quantitatives et qualitatives de la demande. Commercialisant sur un marché très concurrentiel, souvent saturé bien qu'en développement, seules les entreprises dvnamiques qui ont su à la fois améliorer la productivité économique des activités qui dépendaient d'elles,

\section{Résumé}

Ne bénéficiant d'aucune protection communautaire, la production avicole s'est organisée en « filières » spécialisées selon le produit final (œufs de consommation ou viandes de volailles). Maîtres d'œuvre économiques de la production, les entreprises qui mettent en marché les produits finaux, y jouent un rôle prépondérant par la généralisation de l'économie contractuelle. Répondant aux impératifs du marché, ils continuent de la faire évoluer selon les exigences quantitatives et qualitatives de la demande.

Sur le marché communautaire de l'œuf de consommation, une surproduction estimée à $3 \%$ de la production totale a entraîné une chute des prix qui ne se sont rétablis que 3 ans après par la diminution des productions allemande, anglaise et belge.

En France, cette crise a provoqué un tassement de la production, un début de migration de la production vers les concentrations de consommateurs et les régions céréalières et des regroupements nationaux entre d'importants producteurs indépendants.

Le développement spectaculaire de la production et de la consommation des viandes de volailles est expliqué par une amélioration sensible de la productivité de la filière. A l'excellent rapport qualité/prix présenté par les viandes de volailles comparativement à celui des autres viandes, s'ajoute le constant souci des entreprises de transformation de satisfaire les nouvelles habitudes de consommation (découpe, produits élaborés, plats cuisinés). La valeur diététique des viandes de volailles et leur adaptation aux transformations industrielles permettent d'envisager le développement de leur consommation sous de nouvelles formes. et adapter leurs productions aux exigences des consommateurs ont maintenu, voire accru, leur part de marché. Par le jeu d'une économie contractuelle équilibrée elles ont pu développer et diversifier leur production, moderniser leur outil de transformation et de conditionnement tout en demeurant compétitives. Bien que les positions soient maintenant davantage assurées, elles ne sont pas définitivement acquises et comme nous allons le voir, leur maintien nécessite chaque jour un effort supplémentaire d'imagination pour prévoir l'évolution de la demande et mettre opportunément en marché les produits souhaités par les distributeurs et par les consommateurs. Avant que d'analyser, en les replaçant dans le contexte européen, les productions françaises d'œufs de consommation et de viandes de volailles, il ne paraît pas inutile de rappeler quelques généralités qui situent l'importance de l'aviculture dans l'économie agricole nationale.

L'aviculture est la $5^{\mathrm{e}}$ production agricole française avec 20 milliards de francs de livraisons en 1986 soit $7 \%$ du produit total agricole, et la troisième production de viande française avec 1327000 tonnes produites en 1986, après le bœuf (1675 000 tonnes) et le porc (1 591000 tonnes).

Depuis 1980, la France est le premier exportateur mondial de viandes de volailles devant les U.S.A. et le Brésil, avec 327000 tonnes exportées en 1986 soit un chiffre d'affaires en devises fortes de plus de 3 milliards de francs.

En 1986, la consommation nationale a dépassé un million de tonnes de viandes de volailles et près de 14 milliards d'œufs c'est-à-dire, $18,3 \mathrm{~kg}$ de viandes et 265 œufs, soient $33,6 \mathrm{~kg}$ de produits avicoles par habitant et par an. 


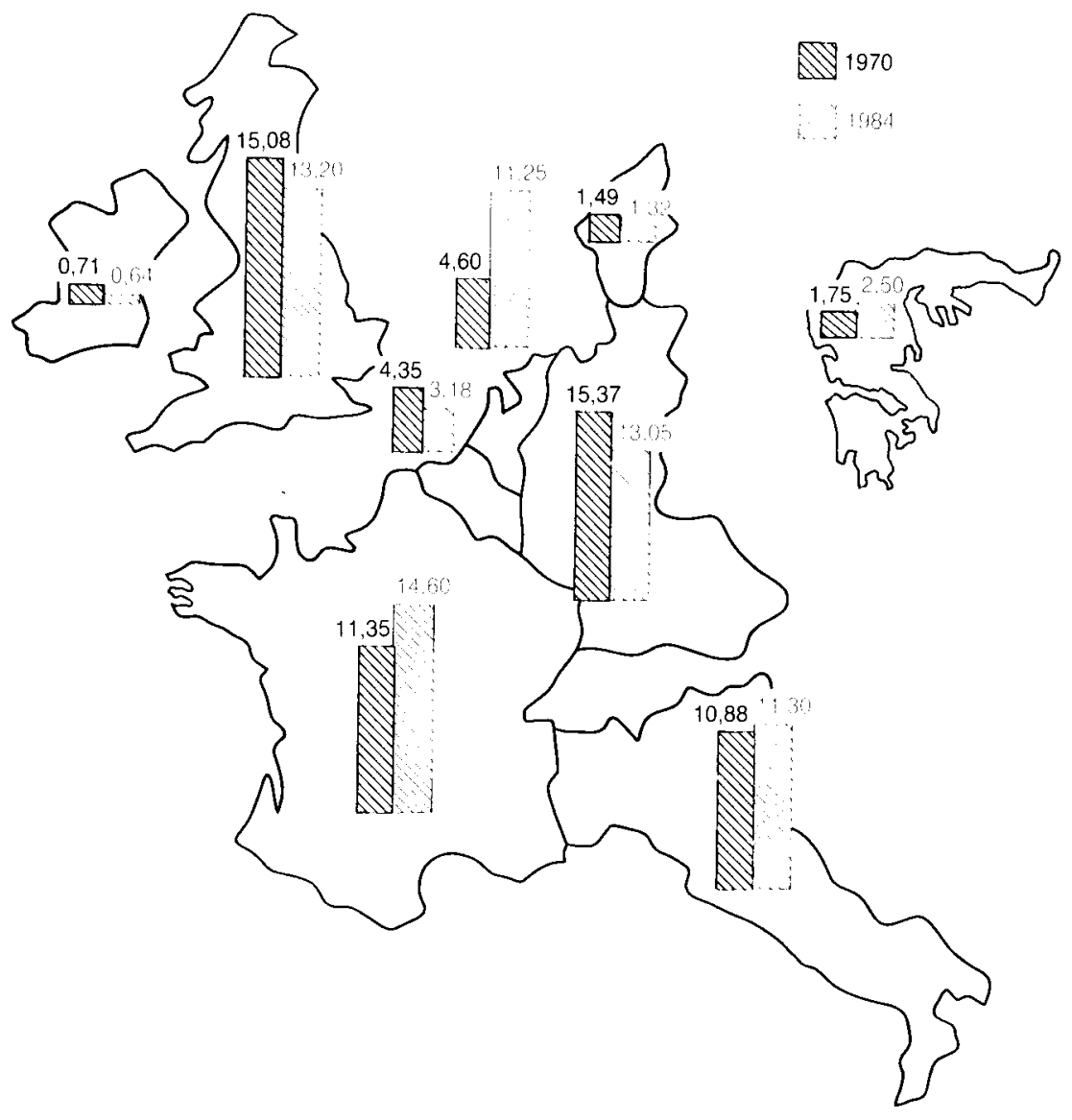

\section{1 / L'œuf de consommation}

\section{1 / Le contexte européen}

\section{a / Consommation}

Entre 1970 et 1984 , la consommation moyenne dans l'Europe des dix a augmenté de $5 \%$ passant de 228 à 239 œufs/habitant/an (Tableau 1). Cette moyenne recouvre des disparités nationales importantes. En effet, si la consommation individuelle a cru sensiblement au Danemark ( $+29 \%)$, en Grèce $(+15 \%)$, en France $(+13 \%)$, en Irlande $(+7 \%)$, et en Italie $(+2 \%)$, elle est restée stable aux Pays-Bas, et a diminué au Royaume-Uni (-14\%), en Belgique, au Luxembourg (-9\%) et en Allemagne (-2\%).

\section{b / Production}

Pour la même période, la production européenne (10 pays) est passée de 65,6 à 71 milliards d'œufs soit une augmentation de $8 \%$ (Figure 1). Cet accroissement, très inégalement réparti, a surtout été le fait des Pays-Bas, qui ont multiplié leur volume de production par 2,5, de la Grèce et de la France qui ont augmenté les leurs respectivement de 43 et de $29 \%$. La production italienne est restée stable $(+4 \%)$ et celle des autres pays partenaires a sensiblement diminué : $-27 \%$ en Belgique, $-15 \%$ en RFA, $-12 \%$ au Royaume-Uni, - $10 \%$ au Danemark et en Irlande.

Figure 1. Production européenne d'œufs de consommation (en milliards d'unités). Source Eurostat-CFCE.

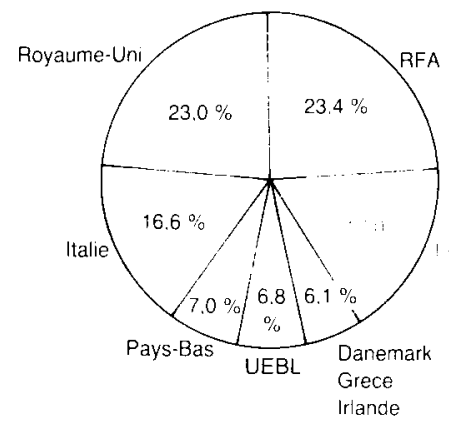

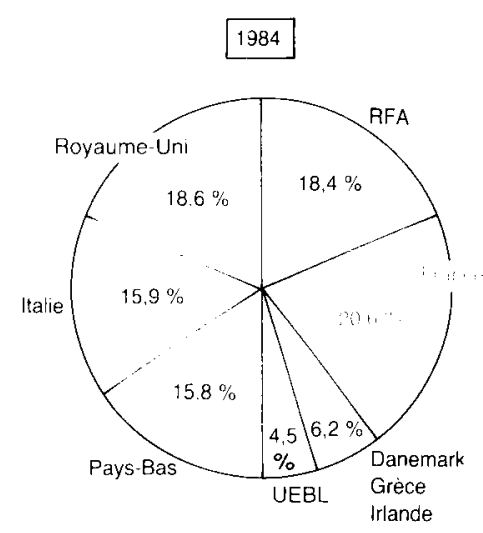

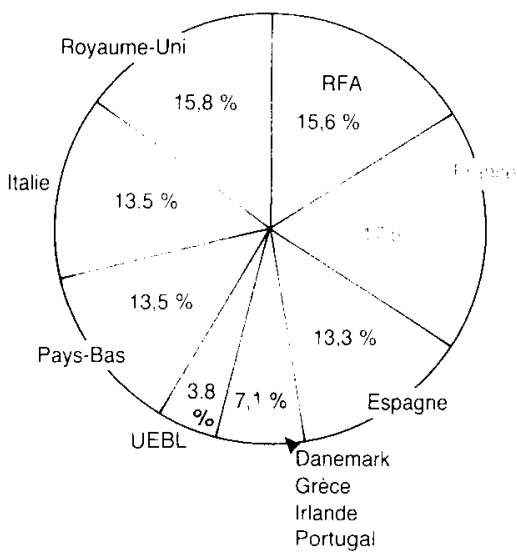

Europe des dix : 71,04 milliards d'unités

Europe des douze : 83,56 milliards d'unités 


\section{c / Marché}

Le marché communautaire se distingue par un échange important d'œufs en coquilles. En effet, la RFA est le plus gros importateur mondial d'œufs (4,7 milliards d'unités importées en 1984 ) et les Pays-Bas le premier exportateur mondial avec 6,2 milliards d'œufs fournis pour la même année. Bien qu'une part importante des expéditions néerlandaises soient destinées à l'Allemagne, considérée comme un marché captif des Pays-Bas, le marché européen est pléthorique, et se trouve dépendant des volumes exportés vers les pays tiers. Or, si jusqu'en 1982 , les soldes du commerce extérieur font apparaître un net développement des exportations, les années suivantes le volume des livraisons aux pays tiers diminue très sensiblement (Figure 2). Sur un marché saturé, la raréfaction des débouchés extérieurs se traduit à partir de 1982 par un effondrement des cours à la production. Ils mettront plus de 3 ans pour revenir à un niveau normal. Ce délai particulièrement long est expliqué par deux raisons différentes :

- la durée du cycle de production d'une pondeuse qui est de 16 mois à partir de la naissance, - la difficulté de prévoir l'ajustement de l'offre et de la demande si l'on y inclut les livraisons extérieures généralement ponctuelles et souvent imprévisibles à moyen terme.

Comme l'illustre particulièrement bien la figure 1, l'accroissement spectaculaire de la production néerlandaise d'œufs de consommation est accompagnée d'une diminution sensible de celle de RFA, du Royaume-Uni et de la Belgique.

Parmi les hypothèses avancées pour expliquer cette évolution, l'une d'entre elles paraît tout à fait vraisemblable :

A partir de 1975, les Pays-Bas incorporent des produits de substitution des céréales importés à bas prix des pays tiers (notamment de Thaïlande) dans l'alimentation des pondeuses. Cet avantage économique ajouté aux facilités portuaires, fluviales et à la forte concentration de la fabrication des aliments de ce pays, permettent une nette diminution du coût de production par rapport à celui obtenu avec un aliment à base de céréales au prix communautaire. S'appuyant sur des structures commerciales particulièrement
Figure 1. Production européenne d'œuts de consommation (en milliards d'unités). Source Eurostat-CFCE.

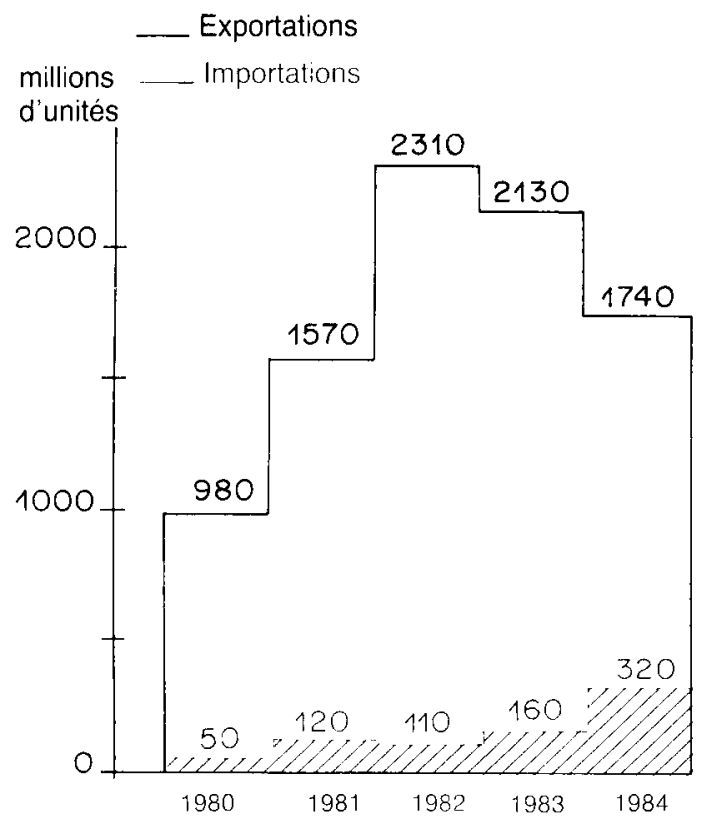

bien adaptées à l'exportation, les producteurs néerlandais accroissent considérablement le volume des œufs destinés à l'exportation notamment à la RFA et aux pays tiers. A partir de 1982, la diminution progressive des envois à destination des pays tiers (Figure 2) provoque un encombrement du marché européen accompagné d'un effondrement des cours et les producteurs les moins compétitifs cessent leur activité. Ainsi s'explique la crise européenne de l'œuf et le tassement de production constaté en RFA, en Grande-Bretagne et en Belgique.

\section{2 / Les particularités nationales}

La consommation et la production françaises d'œufs de consommation ont suivi toutes deux une progression lente mais régulière durant les 20 dernières années. Les soldes annuels du commerce extérieur souvent de faible amplitude (en moyenne autour de $0,5 \%$ de la production)

Tableau 1. Evolution de la consommation d'ceufs par habitant dans la C.E.E. (Europe des dix). Source Eurostat.

\begin{tabular}{|l|ccc|c|}
\hline \multicolumn{1}{|c|}{ Pays } & \multicolumn{3}{|c|}{$\begin{array}{c}\text { Consommation d'oufs } \\
\text { (unités/habitant/an) }\end{array}$} & $\begin{array}{c}\text { Consommation totale } \\
\text { (en milliards d'unité) } \\
\end{array}$ \\
\hline RFA & $\mathbf{1 9 7 0}$ & $\mathbf{1 9 8 4}$ & $\mathbf{8 4 / 7 0}$ & $\mathbf{1 9 8 4}$ \\
France & 281 & 274 & 0,98 & 16,96 \\
Italie & 225 & 255 & 1,13 & 14,30 \\
Pays-Bas & 200 & 204 & 1,02 & 12,27 \\
UEBL & 203 & 204 & 1,00 & 5,22 \\
Royaume-Uni & 263 & 240 & 0,91 & 2,94 \\
Irlande & 275 & 237 & 0,86 & 13,39 \\
Danemark & 216 & 232 & 1,07 & 0,83 \\
Grèce & 178 & 229 & 1,29 & 1,33 \\
\hline Total C.E.E. & 220 & 253 & 1,15 & 2,49 \\
\hline
\end{tabular}


n'indiquent aucune tendance particulière. Ils traduisent souvent un ajustement de l'offre à la demande intérieure.

Cette production est assurée selon deux types différents d'élevage baptisés par le S.C.E.E.S (Service Central des Etudes et Enquêtes Statistiques du Ministère de l'Agriculture) : production intensive d'une part, semi-intensive ou traditionnelle d'autre part. La production intensive correspond à celle des élevages rationnellement gérés et représente, en 1984, $75 \%$ de la production totale. Elle est constituée pour moitié environ par des producteurs groupés autour de centres de conditionnement d'œufs de grosse ou moyenne capacité, en majorité à structure coopérative. L'autre moitié est le fait de producteurs indépendants qui conditionnent euxmêmes leurs produits et les commercialisent directement aux distributeurs voire aux consommateurs. La taille de leur entreprise est fonction du débouché qu'ils ont et peut aller de 5000 à 500000 poules pondeuses par atelier. Dispersés sur l'hexagone, à proximité des centres de consommation, ces producteurs possèdent des installations mécanisées qui leur permettent une diminution sensible des frais de conditionnement et d'acheminement de leurs oufs. S'ils produisent des céréales ou des protéagineux (pois, par exemple), la fabrication " à la ferme " des aliments destinés à leurs poules à partir de ces matières premières permet également une diminution non négligeable du coût de production de l'œuf (le coût de l'alimentation représente environ $65 \%$ du prix de revient de l'œuf). Ces avantages expliquent le début de migration constaté dans la production intensive de l'œuf qui se traduit par une diminution relative de la production bretonne au profit d'autres régions : Midi-Pyrénées, Ile-de-France, Provence-Côted'Azur, Picardie, Normandie, Lorraine, Alsace, Champagne, etc.

La crise a eu pour autre effet le regroupement sous 5 marques nationales de grands producteurs indépendants dispersés dans les différentes régions. Ces 5 groupes (Mas d'Auge, Mâtines, APRO, OPA et "l'œuf de nos villages ») comptent en moyenne 10 éleveurs chacun et totalisent ensemble environ 10 millions de pondeuses soit une production annuelle supérieure à 2 milliards d'œufs de consommation. Ces marques nationales font l'objet de campagnes de publicité et d'opérations de marketing importantes. Elles développent une politique de qualité à partir de cahiers des charges qui définissent avec précision les conditions de production, de conditionnement et de distribution nécessaires pour obtenir et préserver jusqu'à la distribution au détail, des œufs d'une qualité irréprochable. Ces initiatives, complétées par un service de merchandising sophistiqué mis à la disposition des distributeurs ont pour objet de promouvoir l'image d'un œuf de grande qualité commercialisé sous marque. La revalorisation du produit exige qu'il soit sorti de l'anonymat.

Parallèlement à ce type d'actions, certains producteurs ont pris l'initiative de marquer à l'aide d'une encre alimentaire, la date de ponte sur la coquille de l'œuf. Le but de cette opération, contraire à la législation en vigueur, est de garantir auprès du consommateur la fraîcheur de leurs produits. Son succès implique un système de livraison particulièrement rapide. Enfin, sous la pression des pays de l'Europe du Nord, la réglementation communautaire autorise maintenant la mention sur les emballages du mode d'élevage des pondeuses. En conséquence, les consommateurs nostalgiques de la « prairie» ont maintenant la possibilité d'acheter des œufs « issus de poules élevées en plein air $»$.

Ces initiatives prouvent le dynamisme qui anime les responsables de cette production. La diversification des arguments commerciaux est maintenant une réalité et nul doute qu'à l'avenir elle se développe encore.

Face à cette production intensive, il existe toujours une production semi-intensive ou traditionnelle qui représente encore en 1984, près de $25 \%$ de la production totale, soit près de 4 milliards d'œufs. Il s'agit pour partie $(1,2$ milliard d'œufs) d'une production de basse-cour traditionnelle dont les produits sont en grande partie auto-consommés. Le solde, soit 2,6 milliards d'œufs, est produit par de petites unités dispersées sur l'ensemble du territoire et dont la production est en majorité commercialisée directement aux consommateurs, soit à la ferme, soit sur les marchés. L'ensemble de ces productions, bien qu'en diminution constante, bénéficiera sans doute encore longtemps de la préférence des consommateurs notamment auprès des agglomérations de moyenne et de faible importance.

\section{3 / Les ovo-produits}

La présentation des produits d'œufs a peu varié et comporte toujours essentiellement : la coule fraîche, les entiers, les blancs, les jaunes, salés, sucrés, frais, congelés ou déshydratés. Soixante-dix casseries traitent annuellement environ 1,5 milliard d'œufs qui représentent 90000 tonnes d'ovo-produits.

Alors que nous exportons traditionnellement des jaunes en Italie, il semble que les industriels français de l'agro-alimentaire fassent de plus en plus appel aux ovo-produits belges et néerlandais car le solde de notre balance commerciale fait apparaître dans ce domaine un bilan négatif qui est passé de 5800 tonnes (soit 12 millions de francs) en 1980 à 11200 tonnes (75 millions de francs) en 1984

On note quelques innovations destinées à la restauration collective (œufs durs écoqués, saucisson d'œufs durs) et l'apparition timide de plats cuisinés (quiches, soufflés, omelettes).

\section{4 / Conclusion}

Après un développement rapide et important de la part néerlandaise, on assiste depuis 1980 à une stabilisation de la production de l'ensemble des pays de la CEE. Le volume du commerce international de l'œuf en coquille demeure faible et ne laisse espérer aucun développement important. Face une situation difficile, la production française évolue vers des groupements nationaux d'élevages importants à proximité des concentrations de consommateurs et de préfé- 


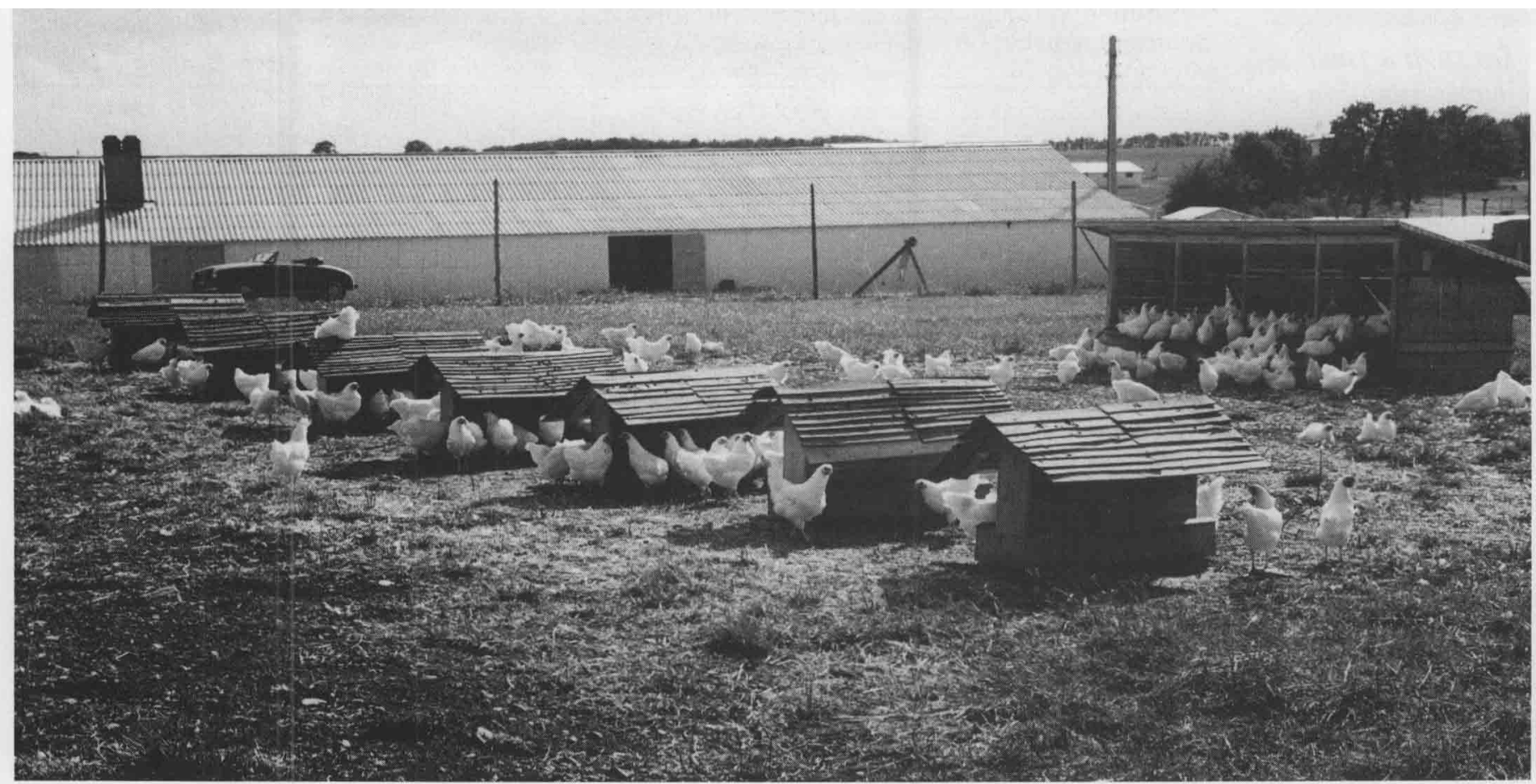

rence en régions céréalières. Ces groupes organisent et diversifient leurs politiques technique et commerciale en vue de satisfaire les différents créneaux qualitatifs offerts par le marché des œufs. Alliant la réduction des coûts de production et de distribution à un réel effort de qualité, il est vraisemblable que leur exemple sera suivi et que la part de ce type de production ne fera que croître à l'avenir. Les contraintes qui caractérisent le marché international des œufs en coquille rendent peu probable un développement important des exportations.

\section{2 / Les viandes de volailles}

\section{1 / Le contexte européen}

\section{a / Consommation}

Entre 1970 et 1984 , la consommation moyenne communautaire de viande est passée de 75 à $90 \mathrm{~kg} / \mathrm{habitant}$ et par an, soit un accroissement de $20 \%$. Dans le même temps, la consommation des viandes de volailles s'est accrue de $65 \%$, passant de 8,8 à $14,5 \mathrm{~kg} /$ habitant et par an (tableau 2). Faible en Allemagne, cet accroissement de consommation est pour les autres pays d'autant plus marqué que la consommation initiale de viandes de volailles était faible (Danemark, Pays-Bas, UEBL).

\section{b / Production}

Pour la même période, la production européenne des 10 pays est passée de 2,6 à 4,3 millions de tonnes, soit un accroissement de $62 \%$, très inégalement réparti (figure 3). En effet, si la Grèce et la France ont pratiquement doublé leur production, les autres pays partenaires ont eu des taux de développement nettement moins importants qui se sont échelonnés de $70 \%$ pour

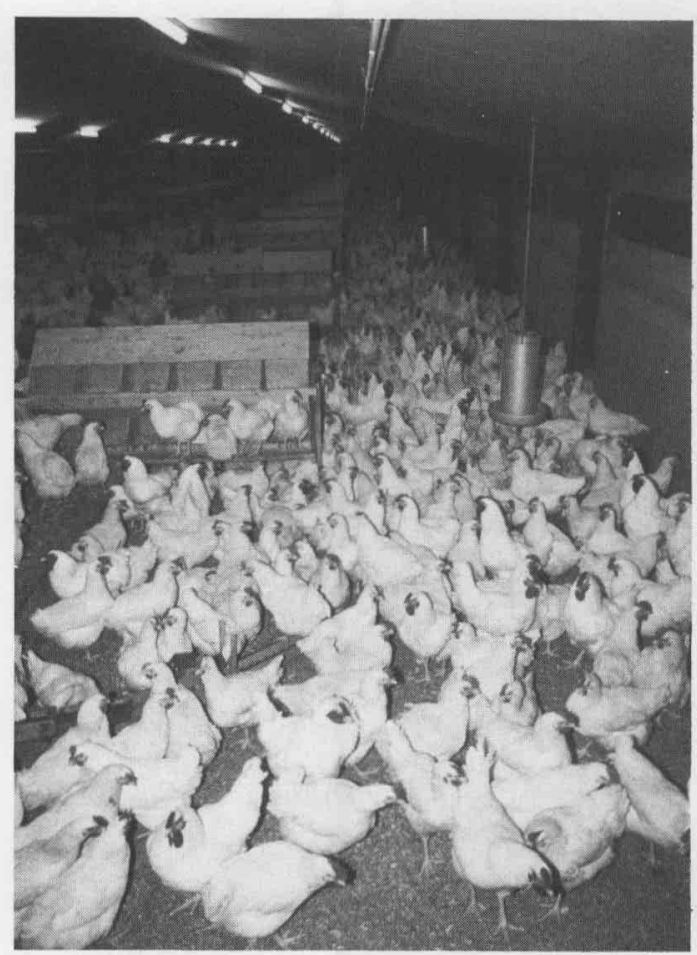

La concentration et la spécialisation des élevages permettent de diminuer les côtts de production.
Poulailler de reproduction au sol.

l'Italie à $11 \%$ pour l'union belgo-luxembourgeoise. A noter, comme le montre le tableau 3, que la part de viande de dinde a crû rapidement et représente, en 1984, $17 \%$ du total des viandes de volailles produites. L'ensemble des autres volailles (poules pondeuses et reproductrices de réforme, canards, pintades, oies) ne représente que $16 \%$ du total des viandes de volailles produit dans la communauté. 


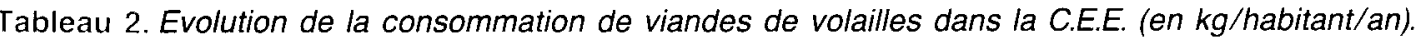
Source Eurostat.

De 1970 à 1984 , la consommation de viande dans la CEE a augmenté de $20 \%$. Dans le même temps, celle de viandes de volailles a augmenté de $65 \%$.

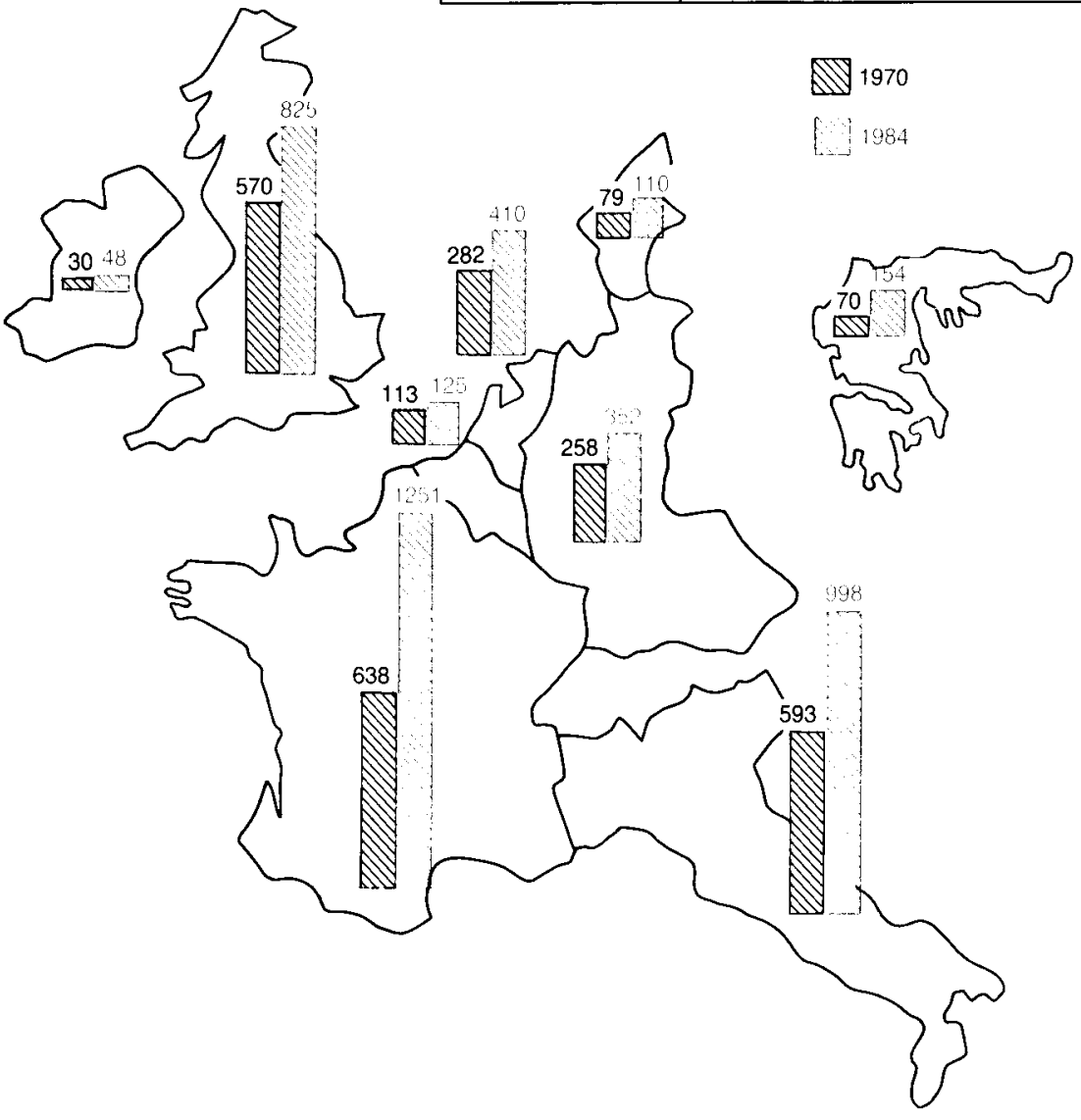

Figure 3. Production européenne de volailles (en milliers de tonnes). Source Eurostat.

\begin{tabular}{|l|cc|cc|c|}
\hline \multicolumn{1}{|c|}{ Pays } & $\begin{array}{c}\text { Total } \\
\text { viandes }\end{array}$ & $\begin{array}{c}\mathbf{1 9 7 0} \\
\text { dont viandes } \\
\text { de volailles } \\
\text { (en \%) }\end{array}$ & $\begin{array}{c}\text { Total } \\
\text { viandes }\end{array}$ & $\begin{array}{c}\mathbf{1 9 8 4} \\
\text { dont viandes } \\
\text { de volailles } \\
\text { (en \%) }\end{array}$ & $\begin{array}{c}\mathbf{1 9 8 4 / 1 9 7 0} \\
\text { Viandes } \\
\text { volailles } \\
\text { \% }\end{array}$ \\
\hline RFA & 82 & 10,2 & 98 & 9,7 & $+13,1$ \\
France & 94 & 12,9 & 105 & 16,5 & $+43,0$ \\
Italie & 54 & 20,4 & 81 & 22,0 & $+61,8$ \\
Pays-Bas & 61 & 9,7 & 80 & 14,6 & $+98,3$ \\
UEBL & 80 & 10,9 & 97 & 15,5 & $+72,0$ \\
Royaume-Uni & 71 & 14,6 & 79 & 19,0 & $+44,2$ \\
Irlande & 81 & 12,3 & 97 & 15,8 & $+53,0$ \\
Danemark & 62 & 7,9 & 80 & 12,9 & $+110,2$ \\
Grèce & & & 78 & 19,9 & - \\
\hline C.E.E. & 75 & 11,7 & 90 & 16,1 & $+64,8$ \\
\hline
\end{tabular}

\section{c / Marché}

Le bilan communautaire des viandes de volailles (figure 4) fait apparaître un net accroissement des exportations qui depuis 1980 représentent $7 \%$ de la production communautaire totale. Ces données ne concernent que les livraisons à destination des pays tiers. Il faut y ajouter les échanges intracommunautaires qui ont porté sur environ 300000 tonnes en 1984. Dans ce total, les livraisons des Pays-Bas à la RFA ont légèrement dépassé les 200000 tonnes.

\section{2 / Le contexte national}

Les viandes de volailles sont la $3^{\mathrm{e}}$ viande française avec 1,31 million de tonnes produites en 1986 , soit $25,5 \%$ de la production totale française de viande (figure 5).

Cette production se répartit en 6 espèces différentes de volailles (tableau 4) à savoir: les poulets qui demeurent l'espèce principale avec $59 \%$ du total en 1986, les poules et coqs de réforme, dont le tonnage est fonction de l'évolution de la production des œufs de consomma-

$1984: 4273$ milliers de tonnes

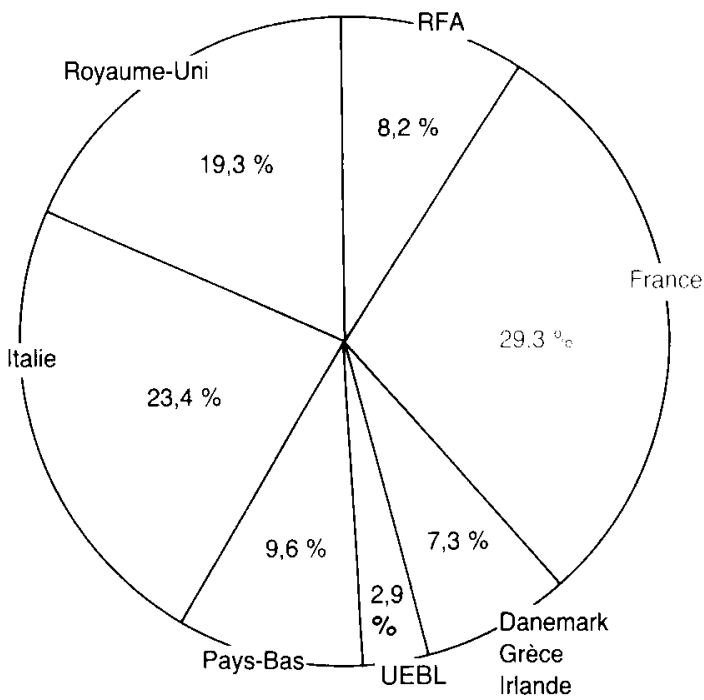


tion et de celle des œufs à couver, les dindes dont le volume depuis 1970 a été multiplié par 10 pour atteindre près de 300000 tonnes en 1986, les canards qui depuis l'introduction du canard de Barbarie sur le marché ont plus que doublé le volume produit, la pintade qui après avoir doublé le tonnage livré plafonne entre 40 à 45000 tonnes par an et les oies qui représentent annuellement environ 2500000 oiseaux dont 1 million de sujets gavés et 1,5 million d'oies à rôtir.

En 1986 (tableau 5), 76,4\% du total des viandes de volailles produites sont destinés au marché intérieur, soit plus d'un million de tonnes ; cette quantité est constituée de $66 \%$ de poulets, $22 \%$ de dindes, $7,3 \%$ de canards, $3,9 \%$ de pintades et $1,2 \%$ d'oies.

Sur le total exporté, les livraisons à destination des pays de la C.E.E. ont représenté environ $20 \%$ des poulets et $80 \%$ des dindes.

Le développement spectaculaire de la production, de la consommation et des exportations des viandes de volailles en France est dû à de nombreuses raisons. Les plus importantes nous paraîssent être les suivantes.

\section{a / L'amélioration permanente de la productivité}

Elle est consécutive notamment :

- aux économies d'échelle liées à la concentration de la production, à la spécialisation et à la
Figure 4. Evolution du bilan communautaire des viandes de volailles.

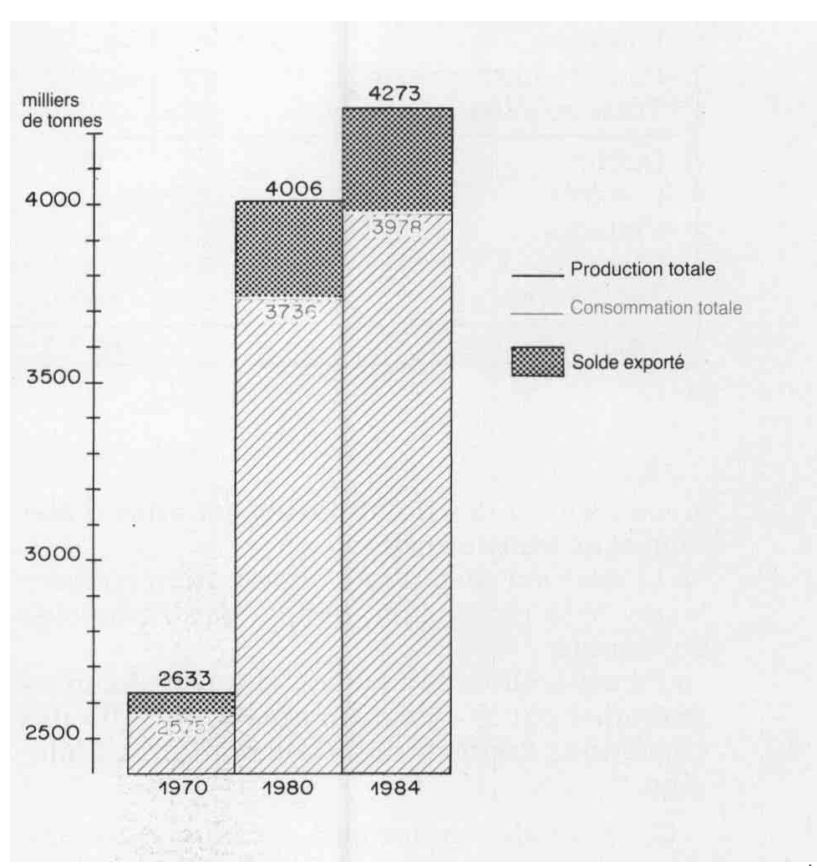

Tableau 3. Répartition de la production communautaire des poulets et des dindes (en milliers de tonnes). Source Eurostat.

\begin{tabular}{|l|ccc|ccc|}
\hline & \multicolumn{3}{|c|}{$\mathbf{1 9 7 6}$} & \multicolumn{3}{c|}{$\mathbf{1 9 8 4}$} \\
\cline { 2 - 7 } & $\begin{array}{c}\text { Total } \\
\text { volailles }\end{array}$ & $\begin{array}{c}\text { Part } \\
\text { poulets } \\
(\%)\end{array}$ & $\begin{array}{c}\text { Part } \\
\text { dindes } \\
{[\%]}\end{array}$ & $\begin{array}{c}\text { Total } \\
\text { volailles }\end{array}$ & $\begin{array}{c}\text { Part } \\
\text { poulets } \\
(\%)\end{array}$ & $\begin{array}{c}\text { Part } \\
\text { dindes } \\
(\%)\end{array}$ \\
\hline RFA & 304 & 68 & 7 & 352 & 62 & 16 \\
France & 865 & 62 & 14 & 1251 & 62 & 20 \\
Italie & 851 & 58 & 20 & 998 & 56 & 23 \\
Pays-Bas & 341 & 86 & 4 & 410 & 82 & 4 \\
UEBL & 106 & 87 & 5 & 125 & 86 & 3 \\
Royaume-Uni & 732 & 67 & 13 & 825 & 75 & 17 \\
Irlande & 52 & 79 & 13 & 48 & 88 & 21 \\
Danemark & 97 & 81 & 3 & 110 & 82 & 3 \\
Grèce & 96 & 95 & 2 & 154 & 82 & 2 \\
\hline C.E.E. & 3444 & 68 & 13 & 4273 & 67 & 17 \\
\hline
\end{tabular}

$1965: 3363$ milliers t.

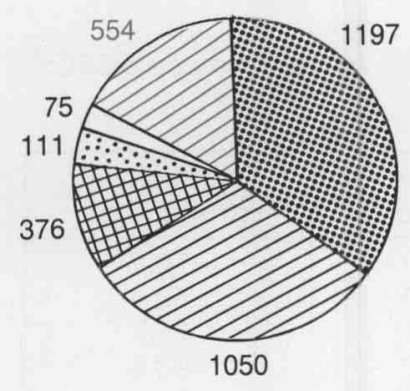

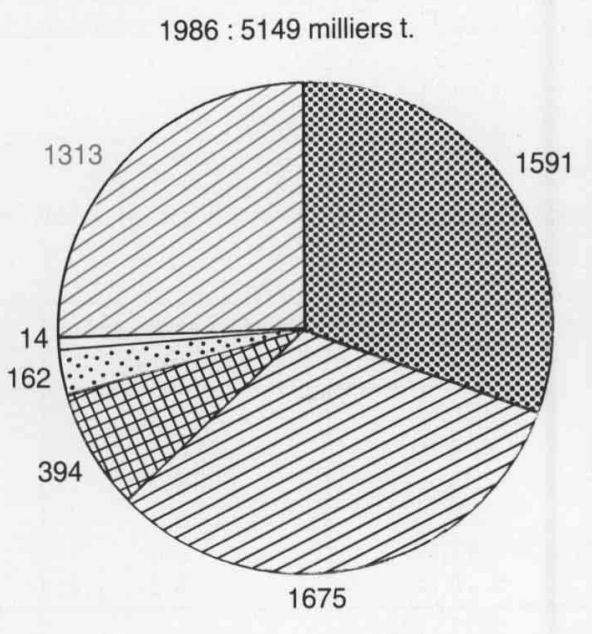

Figure 5. Evolution des différentes productions de viande en France (en milliers de tonnes). 
Tableau 4. Evolution de la production française de viandes de volailles (en milliers de tonnes équivalent carcasse). Source SCEES.

\begin{tabular}{|l|cc|cc|cc|}
\hline \multirow{2}{*}{} & \multicolumn{2}{|c|}{1970} & \multicolumn{2}{c|}{$\mathbf{1 9 8 0}$} & \multicolumn{2}{c|}{$\mathbf{1 9 8 6}$} \\
\cline { 2 - 7 } & Volume & \% & Volume & \% & Volume & $\%$ \\
\hline Poulets & 427,8 & 67,1 & 687,3 & $\mathbf{6 1 , 3}$ & 784,0 & 59,1 \\
Poules - Coqs réformés & 102,2 & $16, \mathbf{0}$ & $\mathbf{1 1 9 , 1}$ & 10,6 & 114,7 & 8,6 \\
Total volailles principales & 530,0 & 83,1 & 806,4 & 71,9 & 898,7 & 67,7 \\
\hline Dindes & 33,2 & 5,2 & 203,3 & 18,1 & 293,3 & 22,1 \\
Canards & 38,9 & $\mathbf{6 , 1}$ & 48,2 & 4,3 & 80,2 & 6,0 \\
Pintades & 21,7 & 3,4 & 45,6 & 4,1 & 42,9 & 3,2 \\
Oies & 13,7 & 2,2 & 18,0 & 0,2 & $\mathbf{8 , 8}$ & 0,7 \\
Total autres & 107,5 & 16,9 & 315,1 & 28,1 & $\mathbf{4 2 5 , 2}$ & 32,0 \\
\hline Total volailles & $\mathbf{6 3 7 , 5}$ & $\mathbf{1 0 0 , 0}$ & 1121,5 & 100,0 & 1326,9 & 99,7 \\
\hline
\end{tabular}

mécanisation des nombreuses activités d'élevage et de transformation,

- à la mise sur pied d'une organisation contractuelle de la production, définie par les besoins du marché,

- à l'amélioration permanente des performances animales par la sélection ainsi que celle des conditions zootechniques et sanitaires d'élevage.

Cet ensemble de facteurs techniques, économiques, sanitaires a considérablement amélioré les rapports qualité/prix des produits.

\section{$b$ / La diversification et la présentation des produits}

Les responsables d'entreprises de transformation ont su, tant à l'exportation que sur le marché intérieur, mettre en valeur les avantages des viandes de volailles, à savoir :

- la diversification : 6 espèces de volailles représentent près de 20 types de produits différents qui, pour chaque espèce, composent une gamme allant du produit standard aux volailles sous label.

Depuis 1970, la production française de dindes a été multipliée par 10 et représente, en 1986 , $22 \%$ de la production de viandes de volailles.
- la facilité d'emploi des produits avicoles qui recouvre :

- les lieux et facilité d'achat : près de $60 \%$ des achats de viandes de volailles sont réalisés dans des surfaces modernes de vente,

- la présentation des produits mieux adaptée aux contraintes de la vie moderne. La part des morceaux et des préparations commercialisés augmente rapidement. En 1985, elle représentait $87 \%$ du tonnage de dinde, $38 \%$ de celui du canard et $20 \%$ de celui du poulet.
- la souplesse d'utilisation industrielle des viandes de volailles : qu'il s'agisse de morceaux, ou des viandes fines récupérées après broyage des carcasses découpées, tous présentent une très bonne adaptabilité à la cuisson, au saumurage, à la déshydratation, aux préparations charcutières, etc.

- l'excellent positionnement des produits de volailles par rapport aux autres viandes. Les différents rapports qualité/prix des produits avicoles bénéficient toujours d'une comparaison avantageuse avec ceux des autres viandes.

- la valeur diététique des viandes de volailles pauvres en graisses et particulièrement digestes ; ces deux particularités correspondent opportunément aux goûts des consommateurs.

La créativité de certains et les efforts permanents de tous se traduisent par un rayon " volaille » qui compte plus de 120 références, toutes d'un prix très abordable. Une telle diversité vise à satisfaire le mieux possible les demandes de l'ensemble des consommateurs, qu'elles soient inspirées par des considérations organoleptiques (avec les produits « haut de gamme » à préparer traditionnellement) ou liées à la commodité d'emploi tel le plateau repas complet pour personnes seules.

Dans le domaine de la sélection de l'ensemble des espèces de volailles destinées à la production intensive de viande (poulet, dinde, canard, pintade), on assiste depuis quelques années à une diversification des critères pris en compte. Le poids maximum à un âge donné, qui a longtemps été le principal caractère sélectionné, est maintenant accompagné de critères de composi-

Tableau 5. Marché français des viandes de volailles en 1986 (en milliers de tonnes). Source SCEESCFCE.

\begin{tabular}{|l|c|c|c|c|}
\hline & Production & $\begin{array}{c}\text { Solde commerce } \\
\text { extérieur }\end{array}$ & $\begin{array}{c}\text { Marché } \\
\text { intérieur }\end{array}$ & $\begin{array}{c}\text { Part } \\
\text { production }\end{array}$ \\
\hline Viandes de volail. & 1326,9 & 351,2 & 1014,0 & 76,4 \\
Poulets & 784,0 & 318,4 & 465,6 & 59,4 \\
Dindes & 293,3 & 68,2 & 225,1 & 76,7 \\
Canards & 80,2 & 6,2 & 74,0 & 92,3 \\
Pintades & 42,9 & 3,6 & 39,3 & 91,6 \\
Oies & 8,8 & $-3,2$ & 12,0 & 136,0 \\
\hline
\end{tabular}


tion corporelle (état d'engraissement, répartition des masses musculaires, etc.) de fertilité, qui visent à la fois des améliorations qualitatives des produits finaux ainsi que la prolificité des croisements commerciaux. Sans minimiser l'influence du prix sur la motivation d'achat des consommateurs, cette constatation témoigne du souci qu'ont les sélectionneurs d'améliorer les caractéristiques du produit final dans le sens souhaité par les consommateurs.

\section{3 / Perspectives \\ de développement des viandes de volailles}

Compte tenu de leurs spécificités, il faut considérer séparément l'avenir de la filière « exportation » et celui de la production destinée au marché intérieur.

\section{a / La filière « exportation "}

La demande internationale de poulets est basée sur un produit standardisé : la carcasse éviscérée avec ou sans abats, de faible poids (entre 900 et $1100 \mathrm{~g}$ ) et présentée congelée. Cette normalisation a amené trois entreprises françaises de très grande taille à organiser une filière totalement intégrée pour en assurer la production dans des conditions économiques optimum. Avec près de 6 millions de poulets par semaine, ces trois firmes assurent $90 \%$ du volume exporté. Toutefois, bien des facteurs totalement extérieurs à l'aviculture conditionnent l'exportation des viandes de volailles. Ainsi, le cours du pétrole, celui du dollar américain, celui des matières premières, les contextes politiques nationaux et internationaux ont une incidence directe sur les volumes importés par de nombreux pays, sur les conditions financières ou la facilité des transactions. Bien qu'avec des difficultés de tous ordres, il est néanmoins prévisible que durant de nombreuses années encore, le volume du commerce international des viandes de volailles augmentera, car la demande existe et de nombreux pays ne peuvent encore la satisfaire malgré les efforts qu'ils déploient pour développer leur production.

Cet ensemble d'éléments complique singulièrement la tâche des exportateurs qui, outre la gestion de la filière de production et de transformation dont ils ont la charge, doivent en permanence assurer leurs marchés, et en prospecter de nouveaux. Conscientes de ces problèmes, ces entreprises font actuellement un gros effort pour diversifier la gamme de leur production (découpe, présentation en containers, etc.) afin d'adapter une partie de leurs présentations aux exigences des pays de la communauté dans laquelle depuis quelques années ils ont réussi à faire une percée commerciale qui se développe.

D'après les résultats actuels, on peut donc espérer qu'à moyen terme le volume des produits exportés dans les pays tiers ou chez nos partenaires devrait se maintenir, voire progresser. A ces expéditions de produits «standards» devraient s'ajouter un volume plus faible mais non négligeable de produits élaborés, conçus pour le marché intérieur, mais appréciés aussi par un nombre croissant de consommateurs européens.

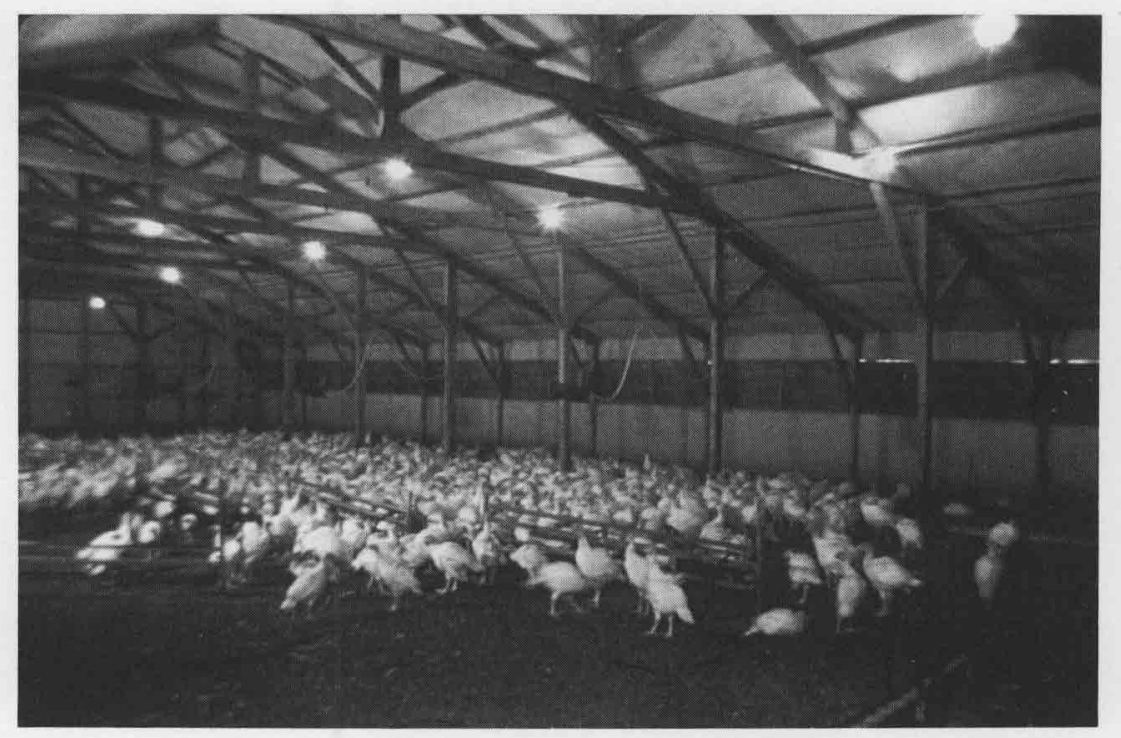

Elevage de dindonneaux.

\section{b / Le marché intérieur}

En ce qui concerne le marché français, la croissance modérée de la consommation constatée depuis 1980, devrait se poursuivre, partagée entre les produits «festifs » de haut de gamme et les préparations commodes d'emploi et de faible portionnement. Pour assurer ces dernières, de nombreuses entreprises de moyenne capacité, qui pour l'instant ne pratiquent que la découpe, devront avoir recours aux activités d'un atelier spécialisé intégré ou contractuel. L'évolution rapide de ces présentations nécessitera un contrôle plus poussé des conditions de production pour obtenir les caractéristiques hygiéniques et technico-économiques demandées par le marché. Une fois de plus, ce sont les entreprises qui maîtrisent avec rigueur tous les aspects de l'élaboration et de la transformation des produits avicoles et qui sauront faire preuve de souplesse et d'innovation dans la mise au point de nouveaux produits qui accroîtront leur part de marché. Comme c'est déjà le cas pour la filière "dinde », la préparation des produits avicoles nécessitera à l'avenir une activité supplémentaire dans laquelle l'imagination et les talents culinaires exercés dans un cadre industriel seront les éléments déterminants de l'accroissement des ventes.

\section{Références bibliographiques}

Publications statistiques des organismes suivants :

- Ministère de l'Agriculture

Direction des Affaires Financières et Economiques Service Central des Etudes et Enquêtes Statistiques 4, avenue de Saint-Mandé - 75570 Paris Cedex 12

- Centre Français du Commerce Extérieur

10, avenue d'léna - 75783 Paris Cedex 16

- Eurostat

L2920 Luxembourg 